\title{
Lidil
}

Revue de linguistique et de didactique des langues

\section{Écrire pour comprendre l'expérience de stage}

Intérêts et limites d'un dispositif numérique collaboratif

\section{Renáta Varga}

\section{(2) OpenEdition}

Journals

Édition électronique

URL : http://journals.openedition.org/lidil/27

DOI : $10.4000 /$ lidil. 27

ISSN : 1960-6052

Éditeur

UGA Éditions/Université Grenoble Alpes

Édition imprimée

Date de publication : 1 décembre 2006

ISBN : 2-914176-15-5

ISSN : $1146-6480$

Référence électronique

Renáta Varga, «Écrire pour comprendre l'expérience de stage », Lidil [En ligne], 34 | 2006, mis en ligne le 07 avril 2008, consulté le 30 avril 2019. URL : http://journals.openedition.org/lidil/27 ; DOI : 10.4000/lidil.27

Ce document a été généré automatiquement le 30 avril 2019

(C) Lidil 


\section{Écrire pour comprendre l'expérience de stage}

Intérêts et limites d'un dispositif numérique collaboratif

\section{Renáta Varga}

1 Dans le cadre d'un projet de recherche intitulé Pratiques Collectives Distribuées d'Apprentissages sur Internet (PCDAI), mené conjointement par les laboratoires GERIICO et T RIGONE, nous avons mis en place en mars 2005 un dispositif numérique pour l'encadrement des stages et de la rédaction des rapports de stage dans une formation en communication à l'université Lille 3 (Varga, 2006). Cet article questionnera l'objectif principal de ce dispositif numérique : amener les étudiants à approfondir leur réflexion tout au long du stage par la rédaction d'écrits intermédiaires afin de les aider à objectiver leur vécu du terrain. Nous nous intéresserons ainsi aux intérêts et aux limites des outils de rédaction proposés sur la plate-forme PostNuke'. Nous interrogerons, notamment, les pratiques et les représentations des étudiants concernant les outils Wiki et Journal de bord (JDB), disponibles en ligne. Enfin, nous chercherons à mettre en lien ces considérations avec la perception qu'ont les étudiants de la démarche de réflexion à mener pendant le stage et des attendus du rapport.

2 La rédaction des écrits longs de type rapport de stage pose de nombreuses difficultés aux étudiants. Plusieurs travaux en didactique en témoignent (Guigue-Durning, 1995 ; Dabène et Reuter, 1998 ; Boch, Laborde-Milaa et Reuter, 2004) et notre expérience pédagogique le montre également. Les problèmes les plus récurrents sont l'identification du genre, l'investissement insuffisant de l'écrivant, une démarche de réflexion inadéquate, l'incapacité de prise de distance par rapport à l'expérience vécue et des problèmes liés à la rédaction proprement dite. Il existe une pléthore d'ouvrages sur le rapport de stage pour aider les étudiants, mais la plupart insistent sur l'aspect professionnalisant du stage et même ceux portant sur la rédaction sont centrés sur les règles formelles et non pas sur le processus d'élaboration et de réflexion. Comme le souligne Rozenn Guibert, les étudiants ne perçoivent pas d'emblée la fonction heuristique de ce type d'écrit (Cros, 1998 : 105), d'où la nécessité de mettre l'accent pendant l'encadrement sur le processus d'élaboration et non pas seulement sur le produit fini. Plusieurs chercheurs soulignent 
l'intérêt de la rédaction tout au long de l'expérience dans un objectif d'auto-observation et d'objectivation du vécu (Cros, 1998 ; Quatrevaux, 2002 ; Varga et Blanc, 2003 ; Leclercq, 2005). Il s'agit la plupart du temps d'écrits destinés au scripteur pour comprendre sa propre expérience, se présentant sous des formes variées, nommés « Journal de bord » ou «Carnet de bord ${ }^{2}$ ».

3 À la lumière de ces considérations, il nous a semblé opportun d'axer notre projet PCDAI sur le processus de réflexion et donc sur l'encadrement de la rédaction d'écrits intermédiaires pour aider les étudiants à prendre du recul par rapport à leur expérience en vue de la rédaction du rapport final. Nous présenterons ici notre démarche pédagogique et l'appropriation de cette démarche par les étudiants en nous appuyant sur leurs usages réels. Nous interrogerons par la suite les représentations sur leurs pratiques, les difficultés et les attendus du rapport de stage. Seront exploitées dans cet article les données issues de l'usage de la plate-forme collaborative dédiée au suivi de stages ${ }^{3}$ en 2005. De plus, deux enquêtes permettront de centrer les investigations sur le point de vue des étudiants :

- un questionnaire administré à treize étudiants en septembre 2005, après usage de la plateforme, portant sur l'appropriation des outils proposés et leurs modes de travail ;

- cinq entretiens d'explicitation (Vermersch et Maurel, 1997) réalisés en novembre 2005 avec les utilisatrices les plus actives permettant d'affiner les résultats obtenus par les questionnaires.

4 L'ensemble de ces éléments laisse apparaitre des décalages entre les intentions pédagogiques et les pratiques étudiantes. Ils résultent, d'une part, d'un certain nombre d'obstacles freinant l'usage d'une plate-forme collaborative dans le contexte donné. D'autre part, ils sont révélateurs de contraintes et de tensions vécues par les étudiants émergeant de discours souvent contradictoires. Les résultats très encourageants montrent, en revanche, les intérêts perçus par les étudiants et permettent d'envisager l'adaptation de la démarche pédagogique aux besoins réels avec la visée de modifier les modes d'accès aux savoirs.

Le dispositif numérique proposé

5 La plate-forme pédagogique ici analysée a été mise en place dans le cadre de l'offre de formation de l'UfR Infocom à l'université Lille 3. Le dispositif de formation intègre une UE «Stage » obligatoire, du niveau Licence au niveau Master 2, avec une expérience d'une durée de six semaines à six mois selon les objectifs pédagogiques ${ }^{4}$. Cette plate-forme a été conçue pour accueillir les étudiants suivis par nous-même, issus de tous les niveaux de formation. En 2005, dix-huit étudiants en licence y étaient inscrits et l'ont utilisée du $1^{\mathrm{er}}$ mai au 23 septembre.

6 S'inspirant des travaux menés par des chercheurs de l'université Lille 1 (Leclercq, 2005 ; D'Halluin et Delache, 2006), nous avons intégré à la plate-forme PostNuke ${ }^{5}$ deux outils de rédaction: un Wiki ${ }^{6}$ et un Journal de bord (désormais JDB) '. Les deux offrant des fonctionnalités complémentaires, ils sont destinés à des usages distincts : le JDB à prendre des notes au jour le jour; le Wiki à structurer les idées en proposant des pistes de réflexion appelées «balises». Ces dernières apparaissent sous forme d'interrogations pour amorcer la réflexion. Ces balises récapitulent les attendus du rapport en termes de types de contenu, prévisibles à partir des objectifs pédagogiques visés. Chaque étudiant dispose d'un espace Wiki incluant la liste de ses balises, présentées sous forme de liens 
vers des pages vides à remplir (cf. ci-dessous). Chacun est libre d'ajouter de nouvelles pages et d'organiser ses pages existantes.

Lieu du stage :

Début et fin du stage :

Mission :

Liste des balises :

Mon [Parcours antérieur], mes motivations pour ce stage.

Comment j'ai réussi à [Prendre place] dans l'organisation?

Quelle est mon [Analyse de la structure] ?

Quelle est ma [Mission]? Qu'est ce que je mets en oeuvre pour atteindre mes

objectifs?

Je décris ici le [Déroulement du stage].

Quels sont les [Apports du stage]? Quelles compétences développées? Quels

apprentissages sur le monde professionnel? Quels bénéfices? Quelles déceptions ?

Comment ce stage s'insère-t-il dans mon [Projet professionnel] ?

Exemple d'un espace personnel Wiki avec la liste des balises ${ }^{8}$

7 Lors d'une séance de formation à l'usage des outils de la plate-forme, la démarche de travail attendue a été explicitée aux étudiants. Aussi, nous leur avons laissé la liberté d'utiliser les outils de rédaction, tout en insistant sur l'intérêt de la réflexion tout au long du stage et l'aide que cela pouvait représenter pour la rédaction du rapport. Ils ont été également encouragés à visiter les pages de leurs pairs et à y ajouter des commentaires". Nous avons aussi précisé que nous visiterions régulièrement l'ensemble des contributions en les annotant éventuellement.

Pratiques et finalités des écrits réalisés pendant le stageUsage des outils de rédaction

8 Huit sur les dix-huit stagiaires encadrés en 2005 ont participé régulièrement aux activités de la plate-forme. Ce résultat parait plutôt décevant, mais nous avons pu mettre en évidence un certain nombre de facteurs contextuels qui constituent des obstacles à l'usage de ce dispositif numérique (Varga, 2005). Ainsi, les compétences techniques préalables semblent déterminantes dans l'appropriation des outils. Par ailleurs, l'accès limité au matériel informatique et à Internet Haut Débit sur le lieu de stage et au domicile étudiant freinent également les usages. De plus, le déroulement de certains stages semble incompatible avec le mode de travail proposé, notamment lorsque l'étudiant doit effectuer des déplacements pendant les heures de stage. Enfin, l'obstacle le plus important nous parait être la non-intégration de l'interface numérique au dispositif de formation. En effet, l'usage de la plate-forme ne peut être imposé aux étudiants ni inclus dans l'évaluation de l'UE dans la mesure où nous sommes la seule intervenante de notre UFR à l'exploiter. Ainsi, pour certains étudiants, l'investissement à fournir peut paraitre trop couteux pour un usage finalement limité. Prenant en compte l'ensemble de ces contraintes, l'activité sur la plate-forme en 2005 nous parait satisfaisante. Le tableau cidessous montre en l'occurrence l'usage des outils de rédaction par les huit étudiants réguliers sur le portail. 


$\begin{array}{lccc}\begin{array}{l}\text { Nombre de } \\ \text { commexions } \\ \text { effectuées }\end{array} & \begin{array}{l}\text { Nombre de } \\ \text { pages Wiki } \\ \text { rédigées }\end{array} & \begin{array}{c}\text { Nombre de } \\ \text { pages crées }\end{array} \\ \text { dans le JDB" }\end{array}$

Usage des outils de rédaction en ligne en 2005

outils de rédaction ontété utilisés, certes de façon inéga outils de rédaction ont été utilisés, certes de façon inégale : malgré son ergonomie peu « bienveillante » le Wiki a davantage été exploité que le JBD. Par ailleurs, quatre étudiants ont utilisé le Wiki et le JDB de façon complémentaire, ce qui montre qu'ils leur ont trouvé des finalités distinctes. Parmi les deux outils, le JDB est plus facile d'usage, mais sept étudiants sur les treize enquêtés pensent que le Wiki est facile à utiliser contre seulement trois concernant le JDB que six étudiants ne connaissent pas du tout, ce qui laisse à penser qu'ils ne l'ont jamais testé.

Importance des balises

Les étudiants justifient l'usage du Wiki par l'intérêt représenté par les balises.

Dans le Wiki on répond à des questions précises qui donnent déjà une orientation sur ce qu'il va falloir développer ou des questions auxquelles il va falloir répondre. S'il y a une question qui figure dans le Wiki et qu'on n'a pas du tout abordée dans notre rapport, je pense qu'il y a un manque quelque part, alors que dans le Journal ce n'est pas du tout guidé, donc finalement on raconte sa vie et ce n'est pas toujours forcément constructif (AP).

11 Ce rôle de guidage ${ }^{10}$ semble important pour tous car l'ensemble des étudiants a consulté les balises, même ceux qui ont peu ou pas rédigé en ligne. Ainsi HC déclare à propos de la plate-forme: "Combien de fois j'ai dit à mes camarades qui n'avaient pas ça pour travailler: moi, ça m'aide bien parce que je sais où je vais ». Son propos fait émerger l'angoisse de l'ensemble des étudiants par rapport à une tâche qui leur est peu connue voire inconnue (rédiger un rapport de stage) et qui génère des remises en question en permanence « on a toujours un doute, on a quand même une soutenance, ça contribue à avoir notre année, on ne peut pas se permettre de se planter, c'est justement ça, des balises » (HC). Elle ajoute que les pistes proposées sur la plate-forme n'étaient pas inconnues pour elle, mais qu'elle a été rassurée de savoir qu'elle ne faisait pas fausse route.

Rôles identifiés des écrits intermédiaires

Nous avons interrogé les étudiants sur leur pratique d'écriture en amont de la rédaction du rapport et sur les finalités de cette pratique. Sept sur les treize étudiants déclarent avoir tenu un journal et/ou pris des notes en dehors de la plate-forme pendant leur stage. La plupart évoque l'objectif de garder une trace du déroulement du stage car ils éprouvent des difficultés à s'en souvenir parfaitement. Ils ont utilisé soit le support papier, soit le traitement de texte. ALH dit avoir noté tout ce qu'il ne fallait pas faire sur un cahier car elle aime bien écrire à la main. $C D$ a elle aussi utilisé le support papier, car elle écrit peu sur l'ordinateur. Enfin AP, l'utilisatrice la plus productive sur la plate-forme, a multiplié les supports de prise de notes: outre les neuf pages Wiki et les deux pages créées sur le JDB, elle a utilisé aussi son agenda sur lequel elle a noté tous les jours ses 
rendez-vous et une liste de choses à faire; enfin elle a créé également des fiches Excel pour marquer tous ses contacts. Elle explique que l'année précédente elle n'avait pas procédé de la même façon car elle devait se déplacer souvent pendant son stage et au moment de la rédaction elle a regretté ne pas avoir pris de notes car elle ne se souvenait plus du déroulement exact de ses missions. Cet exemple montre l'importance des attitudes et des motivations dans les modes de travail. En effet, il ne suffit pas de donner la consigne aux étudiants de rédiger un journal de bord, il faut qu'ils en ressentent réellement le besoin.

13 Si l'on questionne la finalité des rédactions tout au long du stage, tous supports confondus, la plupart des étudiants disent avoir voulu garder une trace pour se souvenir $\mathrm{du}$ déroulement exact $\mathrm{du}$ stage. Une étudiante déclare avoir voulu consigner ses premières impressions, une autre voulait organiser ses idées. Deux étudiantes évoquent la mise à plat et la clarification des idées, une seule parle de prise de distance. Cette dernière explique que l'écriture l'aidait à réfléchir parce qu'elle s'est rendu compte qu'elle prenait les choses très à cœur. Par exemple, écrire qu'elle n'allait pas bien, qu'elle «en avait marre » qu'elle était « dégoutée » et pouvoir le relire plus tard, l'aidait à prendre de la distance (AP). Ainsi les étudiants attribuent deux fonctions aux écrits intermédiaires : prioritairement une fonction d'aide-mémoire (garder une trace et des impressions) et secondairement une fonction de réflexion (organiser et clarifier les idées, prendre du recul).

14 Contrairement à la majorité des étudiants qui ont pris des notes sous une forme ou sous une autre, quatre étudiants déclarent ne rien avoir rédigé pendant le stage: deux par manque de temps et deux parce qu'elles se souviennent parfaitement de leur stage : «[...] forcément, ce sont des choses que je connais parce que c'est moi qui les ai vécues [...], je n'ai pas pris de notes pendant le stage parce que j'étais tout le temps très active, j'allais pas oublier de toute façon ce que j'avais fait parce que c'était tellement intéressant [...] » $(\mathrm{HC})$. On peut pointer ici un décalage important entre intentions pédagogiques et représentations étudiantes. En effet, les écrits intermédiaires et notamment les notes prises au jour le jour permettent de mémoriser le déroulement des actions, les impressions vécues, mais leur finalité est de prendre du recul, objectif verbalisé explicitement par une seule étudiante. D'autres considèrent la prise de notes inutile argüant qu'ils possèdent une bonne mémoire. Le rôle de ces représentations n'est pas à négliger car, comme le remarque Yves Reuter, elles peuvent constituer un obstacle à la pratique et à l'apprentissage (1996). Il nous semble donc primordial de pouvoir agir sur ces représentations en proposant une autre interprétation de la démarche pédagogique proposée, ce qui permettrait d'influer sur les modes de travail.

Dissociation des écrits intermédiaires et du rapport final

15 La déclaration de certains étudiants sur le devenir des écrits produits pendant le stage fait apparaitre une rupture entre ces notes et le rapport final. Alors que certains disent que ces écrits ont constitué une base pour la rédaction du rapport, d'autres affirment catégoriquement qu'ils n'ont pas été utiles pour la rédaction finale. Ils citent plusieurs raisons. ALH déclare que ses pages du JDB étaient en réalité destinées aux autres étudiants et à la tutrice, et que c'est la raison pour laquelle elle ne s'en est pas servi pour la rédaction du rapport. Une autre étudiante évoque la divergence de contenu des textes élaborés. Elle a rédigé en effet quelques pages Wiki, mais ne les a pas utilisées pour le rapport car elle s'est rendu compte que "ça ne collait pas » avec son plan (CD). Cette vision est révélatrice de sa méthode de travail qui n'est pas basée sur la réécriture. Les 
notes prises sur la plate-forme n'étant pas formatées pour entrer dans les parties pressenties du rapport, semblent pour elle «inutiles» et «inutilisables» pour la rédaction finale. Ici l'élaboration du rapport est considérée comme une tâche ponctuelle et non pas comme un processus de maturation de la pensée. Nous rencontrons le même type de fonctionnement lorsque certains de nos étudiants déclarent à propos de leur écrit de type réflexif ne pas être satisfaits du produit fini malgré le temps passé à son écriture : $\mathrm{X}$ jours en continu. Selon cette considération, le travail d'écriture se limite à l'opération de scription et l'étudiant sous-estime l'importance des opérations de planification (Reuter, 1996). Dans ce cas, il s'agit bien d'un problème de compétence d'écriture qui, se manifestant par une représentation erronée, ne peut être corrigé par un simple rappel de consignes.

Enfin, le discours de HC qui a rédigé trois pages Wiki révèle une stratégie de rédaction, témoignant également d'un décalage concernant la représentation des écrits intermédiaires. «Sur la plate-forme, à la base, ça ne devait pas être la rédaction de la version finale, c'est une mise en ligne des idées. Moi, je suis quelqu'un qui n'arrive pas à mettre des idées, des flèches [...], c'est pour ça que c'était quand même rédigé et j'ai commencé par ça, et je n'ai pas continué parce que je me suis rendu compte que ce que je mettais, ça pouvait être dans le rapport, c'est pour ça que je suis passée directement sous Word ». Cette étudiante identifie donc les écrits intermédiaires à travers leur aspect formel et non pas à travers leur contenu ou visée. C'est cette forme représentée qui semble être discriminante entre les deux types d'écrits: brouillons (prises de notes)/ rapport de stage (texte rédigé). Cette conception erronée révèle aussi l'importance des normes formelles dans la représentation de ce type d'écrit et son influence sur la compétence d'écriture".

Mais la remarque de $\mathrm{HC}$ soulève aussi un autre problème, cette fois d'ordre technique, que nous avons identifié comme un frein à l'usage : aucun des deux outils de rédaction proposés sur la plate-forme ne permet un transfert facile des données vers un traitement de texte pour récupérer les écrits. Pour migrer ses pages sur Word, il faut copier-coller le contenu de chaque page avec l'inconvénient que le texte transféré doit être épuré du code utilisé dans le Wiki pour la mise en page. Il s'agit pour nous d'un obstacle très important car l'utilisabilité représente un des trois critères essentiels de l'évaluation d'une interface numérique (Tricot et al., 2003).

En fin de compte, les représentations qui se dégagent des discours des étudiants montrent qu'il ne suffit pas de mettre en place des outils de rédaction et d'insister sur l'importance de l'écriture tout au long du stage, mais qu'il est aussi nécessaire de les relier davantage au produit fini « rapport de stage». Un encadrement pédagogique plus directif portant sur la nature des écrits à produire et sur le lien entre ces types d'écrits se révèle être donc une piste intéressante à explorer.

Bilan des étudiants

19 La mise en place d'outils de rédaction sur une plate-forme collaborative permet la construction d'une mémoire collective, nourrie par l'ensemble des contributions proposées. Ainsi, les productions de chacun contribuent à un enrichissement mutuel, bénéfique quelles que soient les orientations et les expériences respectives. Les discours des étudiants à propos de la construction de cette mémoire collective sont traversés par un réseau de contradictions laissant apparaitre des tensions et des angoisses vécues, dépassant le cadre de l'élaboration d'un rapport de stage : ces dernières sont révélatrices 
de leur compétence scripturale (Dabène, 1987). Les modes de fonctionnement ainsi dévoilés sont parfois éloignés des représentations construites par l'enseignant.

Notons que l'enquête révèle également la satisfaction des étudiants à propos du suivi pendant le stage. En effet, la plate-forme collaborative permettrait une relation pédagogique plus proche et plus humaine que lors d'un suivi traditionnel, grâce notamment aux formes d'échanges qu'elle propose.

Intérêt pour la production des pairs

21 Les étudiants déclarent sans exception leur intérêt pour les contributions de leurs pairs. Le nombre important de connexions y compris pour ceux qui ont peu produit sur la plateforme en témoigne (cf. tableau ci-dessus). Ils ont ainsi effectué de nombreuses visites pour prendre connaissance de l'avancement du travail des autres. AP souligne l'enrichissement personnel, même lorsque le stage des autres étudiants était très éloigné de ses propres préoccupations :

Je trouvais ça intéressant, même s'ils faisaient quelque chose qui ne me plaisait pas du tout, par exemple journalisme, C. c'est vraiment quelque chose qu'elle veut faire. Moi, quand j'ai lu, je me suis dit: c'est marrant parce qu'elle est très motivée, autant c'est quelque chose qui ne m'intéressait pas du tout, mais c'est bien de voir ce qui l'intéressait, ce qu'elle faisait [...].

HC insiste sur l'importance de se comparer aux autres : «C'est toujours instructif de voir ce que les autres ont fait et puis pour se situer ». Selon NG, l'intérêt de consulter les productions des pairs est que le fait de ne pas être impliqué personnellement dans leur expérience permet de comprendre les difficultés rencontrées par les autres et de repérer plus facilement les défauts de leurs démarches. Ces travaux permettent alors de relativiser ses propres difficultés, donc de prendre du recul et d'avancer dans sa réflexion. Cet intérêt semble être partagé par la plupart des étudiants pour qui la plateforme a permis de découvrir la multiplicité des situations de stage, mais avec de nombreux dénominateurs communs. La prise de distance se fait donc en miroir, par l'intermédiaire des réflexions des pairs qui deviennent alors supports et facilitateurs de la réflexion. Cependant, il est intéressant de noter que les étudiants semblent s'intéresser davantage aux travaux des amis ou des personnes qui ont généralement de bons résultats, ce qui laisse supposer une certaine méfiance vis-à-vis de leurs pairs'.

Aussi l'ensemble des enquêtés, même ceux qui ont peu rédigé sur la plate-forme déplorent que les autres étudiants n'aient laissé que peu de contributions. Cette contradiction est intéressante à souligner car elle montre la difficulté, voire l'impossibilité de constituer une communauté d'apprentissage ou une communauté de pratiques (D'Halluin et Delache, 2006) dès lors que chacun des membres du portail poursuit un objectif personnel. Ici, le stage et le rapport de stage étant un travail personnel, il est difficile de concevoir pour les étudiants d'effectuer une tâche individuelle tout en collaborant avec les autres ${ }^{13}$. Les étudiants évoquent plusieurs raisons à leur faible production écrite sur le portail.

Réticence à laisser des contributions et à s'entraider

24 La majorité des étudiants enquêtés (7 sur 13) se sent gênée de laisser des contributions à la disposition des autres étudiants. Les raisons invoquées se rejoignent souvent et seulement une stagiaire parle de secret professionnel qui lui est imposé. Les autres évoquent la nature personnelle des informations ou leur crainte d'être jugés. Ainsi, NG justifie le fait d'avoir mis ses pages du Journal en privé ${ }^{14}$ car elle les considère comme contenant des informations personnelles ne concernant pas ses camarades. Elle est aussi 
réticente à laisser des pièces jointes à disposition des autres par manque de confiance et aussi parce qu'elle ne souhaite pas que ses pairs puissent consulter ses brouillons parce que « ce n'est pas forcément quelque chose de très brillant »; «j'ai très bien pu écrire une belle ânerie et je n'ai pas forcément envie que tout le monde la voie, pour qu'ils disent: oh là là, elle est débile! ». Elle exprime ici par ces mots fortement connotés sa crainte d'être jugée. Elle reconnait cependant que les autres étudiants ne sont pas là pour la juger et qu'ils commettent certainement les mêmes erreurs qu'elle. Ces craintes généralisées renvoient à l'insécurité scripturale des sujets (Dabène, 1987 ; Barré-De Miniac, 2000) qui est d'autant plus exacerbée que les étudiants décrivent leur formation comme un univers de compétition. En lien avec cette idée, HC déclare en plaisantant que grâce à la plateforme elle a pu « espionner » ses camarades.

La crainte d'être évalué n'empêche pas de formuler un jugement de valeur vis-à-vis des autres. À ce propos, le discours de AP est intéressant à relever. Elle raconte son indignation par rapport aux faiblesses orthographiques d'une camarade :

[...] j'étais horrifiée, ça m'a choquée, énormément [...] je n'ai jamais travaillé avec elle mais je sais que c'est un problème parce qu'elle écrit comme elle entend, par exemple j'ai accès elle écrit axé. On peut faire des fautes d'orthographe, par exemple ne pas mettre des $s$ ou si on tape vite, ça m'est souvent arrivé en écrivant [...] ça doit être handicapant après pour tout [...] on en a discuté avec N. et on s'est demandé comment les profs n'avaient pas réagi à propos de ça.

Ce type de critique ne nous semble pas isolé, d'autant plus que très récemment nous avons relevé sur la plate-forme le message d'une étudiante avertissant une camarade de ses nombreuses erreurs orthographiques. Ces discours sont à mettre en lien avec l'attachement des étudiants aux règles formelles, révélé dans la partie précédente. Par ailleurs, 9 sur les 57 étudiants de licence questionnés en 2006 (cf. NBP n¹3) mentionnent les problèmes d'orthographe parmi les difficultés de rédaction qu'ils identifient à rédiger un rapport de stage.

Quant à l'idée d'entraide, elle surprend les étudiants qui disent ne pas se sentir compétents pour aider les autres : "Je n'ai jamais fait de commentaire sur le travail de personne [...] parce que j'estime que je n'ai pas les compétences pour dire à quelqu'un d'autre comment on peut faire » (NG). Pourtant, la même étudiante reconnait pouvoir se faire une idée précise sur la qualité du travail réalisé lorsqu'elle consulte les rapports de ses pairs. On peut constater par ailleurs une méfiance vis-à-vis de l'appréciation des pairs : «Peut-être on estime que l'aide d'un autre étudiant n'apportera pas forcément la bonne réponse, ça peut nous induire en erreur, ce n'est pas parce que C. l'a fait que c'est forcément ce qu'il fallait faire [...] » (NG). Cette attitude montre un fonctionnement traditionnel fondé sur une relation hiérarchique entre étudiants et enseignant qui est pourtant modifiée dans un contexte de dispositif numérique où chacun est amené à s'adapter aux règles induites par cette nouvelle forme de relation pédagogique dans laquelle les rôles doivent s'ajuster en fonction des places que prend chacun des acteurs (Choplin et Hotte, 2004 ; Fichez et Varga, 2006).

Bilan positif de l'accompagnement

Malgré les difficultés, les obstacles techniques et le faible nombre de contributions, les étudiants dressent un bilan très positif de cette expérience numérique, notamment en terme d'accompagnement. Ils estiment que la plate-forme a rendu le contact plus facile avec la tutrice qui était disponible pour répondre à leurs questions ou annoter leurs travaux. Ils admettent donc que la plate-forme leur a permis d'approfondir leur 
réflexion; certes leur discours porte essentiellement sur le stage et peu sur la rédaction du rapport. Ils semblent donc dissocier le stage de la rédaction de l'expérience, comme ils dissocient les écrits intermédiaires du rapport de stage.

Les étudiants pensent que l'usage de ce dispositif numérique induit un changement dans la relation avec leur tuteur: ALH parle de plus de proximité et d'humanité. HC souligne, elle, le côté rassurant du dispositif: "[...] c'est plus la possibilité, c'est limite psychologique parce qu'on est rassuré quelque part, parce que je vois que vous êtes là ». Ce que l'on peut en conclure, c'est que, paradoxalement, l'outil informatique, au lieu d'ajouter une distance dans la relation pédagogique, rapproche les acteurs en créant un lien de proximité entre eux. L'éloignement physique est atténué par la rapidité des échanges et par une co-présence potentielle sur la plate-forme.

Par ailleurs, l'espace de travail offert par le dispositif numérique induit une nouvelle forme d'accompagnement qui semblerait convenir aux stagiaires. Ainsi, ils savent que leurs contributions sont consultées par l'enseignante, qui peut faire un retour si elle estime que cela est nécessaire. «[...] quand on met des choses en public ça permet de l'adresser à vous sans forcément l'adresser à vous parce que je ne vous aurais pas envoyé un mail en vous racontant ma journée par exemple, parce que ce n'est pas intéressant comme ça, mais je sais qu'à côté de cela vous avez pu aller le voir et en prendre connaissance quand même » (ALH). Leur travail représente donc un support à un échange potentiel, ce qui les motive pour participer aux activités proposées sur la plate-forme. Ce facteur motivation nous semble très important, car comme Alain Quatrevaux l'a montré sur une expérience d'auto-observation (2002), il joue, avec d'autres facteurs psychologiques, un rôle important dans l'autoformation.

ConclusionBilan positif du dispositif numérique proposé

31 Cet article avait pour objectif de questionner les intérêts et les limites d'une plate-forme pédagogique collaborative, notamment à travers l'usage de deux outils de rédaction pour approfondir la réflexion sur l'expérience de stage. Notre étude montre un usage réel mais limité de la plate-forme, avec un intérêt manifeste pour les outils mis en place, mais des modes de travail différents de ceux prévus par l'enseignante. Ainsi, malgré nos préconisations, les étudiants ont relativement peu rédigé en ligne pendant leur stage ; ils étaient en revanche très désireux de prendre connaissance des écrits de leurs pairs et demandeurs d'un accompagnement soutenu. Ils ont donc perçu les enjeux qu'une mémoire collective représente pour l'autoapprentissage, mais sont encore réticents à une collaboration entre pairs qui leur est inhabituelle dans ce cadre précis.

La première session d'expérimentation de la plate-forme a fait émerger un certain nombre de limites qui restent malgré tout contournables. Pour la plupart, elles sont contextuelles et renvoient aux conditions de la dynamisation d'une formation numérisée (D'Halluin, Delache, 2006). La condition qui nous semble la plus importante est l'intégration de la plate-forme dans le dispositif de formation, ce qui n'est pas le cas dans notre exemple d'usage prototypique avec un groupe restreint. Le facteur temps semble également déterminant dans la mesure où un usage ponctuel de 6 semaines à 3 mois peut décourager les étudiants peu familiers aux NTIC. Notre étude a fait émerger aussi quelques obstacles techniques, liés aux fonctionnalités et à l'ergonomie de la plate-forme utilisée. Enfin, une dernière limite et non des moindres est l'investissement pédagogique exigé car malgré une mutualisation des informations offerte par les caractéristiques du dispositif, le temps d'encadrement est largement supérieur à celui d'un accompagnement traditionnel. 

notamment les outils de rédaction Wiki et JDB ont incité les étudiants à s'investir dans le travail de réflexion tout au long du stage et à produire des écrits intermédiaires, certes en nombre limité. Les stagiaires déclarent être satisfaits pour plusieurs raisons. Ils se sont sentis davantage soutenus et accompagnés que lors d'un suivi traditionnel; de ce fait, le dispositif les a rassurés et motivés à participer aux activités proposées. De plus, la plateforme représentait pour eux un lien potentiel avec le monde universitaire, à un moment où ils en étaient coupés. Enfin, ils soulignent le plaisir d'avoir participé à l'animation du dispositif. Ajoutons que cette motivation et ce plaisir sont pleinement partagés par l'enseignante. En effet, le caractère collaboratif de la plate-forme PostNuke permet une relation pédagogique privilégiée: l'espace numérique rapproche paradoxalement les sujets, restructure les rôles et offre la possibilité au tuteur de s'associer aux joies et aux déceptions de chaque expérience singulière. Notre motivation reste donc intacte dans cette aventure avant tout humaine.

Suite de l'expérience et pistes didactiques cette $2^{\mathrm{e}}$ année d'usage est très positif : les étudiants étaient très assidus ${ }^{17}$ et ont rédigé davantage en ligne ${ }^{18}$. Par ailleurs, leur mode de travail a évolué car en plus de la rédaction en ligne, ils ont déposé sur la plate-forme de nombreux documents de travail réalisés sur un traitement de texte : note d'orientation, guide d'entretien, ébauche de plan, parties du rapport, etc. Ces documents étaient destinés au travail individuel ou à une évaluation sollicitée. Soulignons que les mêmes étudiants qui ont déclaré en 2005 être gênés de communiquer leurs travaux à l'ensemble du groupe ont franchi le pas l'année suivante et ont sollicité même leurs pairs pour un avis. Le climat de confiance a participé certainement à cette évolution. Il nous semble aussi que cela révèle une dynamique spiralaire où la mémoire constituée et l'activité de la plate-forme motivent en retour les étudiants à l'utilisation des outils proposés.

À la lumière de l'expérience de 2005, trois pistes didactiques

Premièrement, il nous semble opportun d'envisager un travail sur les spécificités du module Stage. En effet, comme Michèle Guigue-Durning le remarque, le support de validation du Stage est déconcertant pour les étudiants car il n'est pas conforme aux caractéristiques habituelles des épreuves universitaires (1995). Ainsi, établir clairement les liens entre stage/rapport de stage/soutenance permettra aux étudiants d'envisager plus sereinement le travail attendu. Cela permettra aussi de relier le travail de réflexion pendant le stage à la production de l'écrit final.

Deuxièmement, nous souhaitons centrer notre démarche pédagogique davantage sur le guidage des étudiants. Une analyse des productions étudiantes envisagée à l'automne 2006 permettra de mieux appréhender les limites des activités de rédaction proposées. Cette analyse débouchera sur l'élaboration d'une grille d'observation ciblée sur les objectifs pédagogiques, que nous fournirons aux stagiaires en 2007. Aussi, pour répondre au besoin de guidage plus directif révélé par notre enquête, nous souhaitons proposer un scénario pédagogique ${ }^{19}$ qui permettrait de structurer les activités d'écriture proposées.

Enfin, troisièmement, il nous restera à encourager les collaborations entre pairs, en créant par exemple des groupes de travail entre étudiants intervenant dans des domaines similaires, ce qui impulsera, nous l'espérons, une triple dynamique où les trois logiques 
dispositive, numérique et de groupe participent conjointement à la construction d'une communauté d'apprentissage (D'Halluin, Delache, 2006).

\section{BIBLIOGRAPHIE}

BARRÉ-DE MINIAC, CH. (2000) : Le rapport à l'écriture, Aspects théoriques et didactiques, Lille, Presses universitaires du Septentrion.

BOCH, F., LABORDE-MILAA, I. et REUTER, Y. (coord.), (2004) : Les écrits universitaires, Pratiques, 121-122, Metz, CRESF.

CHOPLIN, H. et HOTTE, R. (coord.), (2004) : Énigmes de la relation pédagogique à distance, Distances et savoirs, vol. 2, 2-3, Paris, CNED/Lavoisier.

CROS, F. (éd), (1998) : Le mémoire professionnel en formation des enseignants, Un processus de construction identitaire, Paris, L'Harmattan.

DABÈNE, M. (1987): L'adulte et l'écriture, contributions à une didactique de l'écrit en langue maternelle, Bruxelles, De Boeck.

DABÈNE, M. et REUTER, Y. (coord.), (1998) : Pratiques de l'écrit et modes d'accès au savoir dans l'enseignement supérieur, Lidil, 17, Université Grenoble 3.

DELACHE, D. et D'HALLUIN, CH. (2005) : Entre pratiques pédagogiques et pratiques d'apprentissage, Effets induits d'un changement d'interface numérique, Colloque SIF « Les institutions éducatives face au numérique ", Paris, 12-13 décembre 2005.

Consultable sur Tematice : < http://archive-edutice.ccsd.cnrs.fr >

DELCAMBRE, I. et REUTER, Y. (coord.), (2002) : Images du scripteur et rapports à l'écriture, Pratiques, 113-114, Metz, CRESEF.

DELCAMBRE, I. et JOVENET, A.-M. (coord.), (2002) : Lire-écrire dans le supérieur, Spirale, 29, Université Lille 3.

D'HALLUIN, CH. et DELACHE, D. (2006) : Étude d'un processus dynamique de construction d'une communauté par interactions entre dispositif et interface numérique, Actes du colloque JOCAIR, 6-8 juillet 2006, Amiens, 51-75.

FICHEZ, E. et VARGA, R. (2006) : Plate-forme collaborative « Postnuke » : dynamiques de coconstruction et co-évolution, Actes du colloque JOCAIR, 6-8 juillet 2006, Amiens, 27-50. GOMEZ, F. (2001) : Le mémoire professionnel, Objet de recherche et outil de formation, Bruxelles, De Boeck Université, 2001.

GUIGUE-DURNING, M. (1995) : Les mémoires en formation, Paris, L'Harmattan.

LE BOTERF, G. (1997) : De la compétence à la navigation professionnelle, Paris : Les éditions d'Organisation. 
LECLERCQ, G. (2005) : Les dispositifs de formation professionnalisés comme situation d'auto et d'inter-confrontation. L'impact d'un environnement numérique de travail, Colloque SIF « Les institutions éducatives face au numérique », Paris, 12-13 décembre 2005,

Consultable sur Tematice : < http://archive-edutice.ccsd.cnrs.fr > MOLINER, P. (dir.), (2001) : La dynamique des représentations sociales, Grenoble, PUG.

QUATREVAUX, A. (2002) : Journaux de formation, analyse de discours et communication orale, Paris, L'Harmattan.

REUTER, Y. (1996) : Enseigner et apprendre à écrire, Paris, ESF éditeur.

STRAUSS-RAFFY, C. (2004) : Le saisissement de l'écriture, Paris, L'Harmattan.

TRICOT, A., PLÉJAT-SONTJIS, F., CAMPS, J-F., AMIEL, A., LUTZ, G. et MORCILLO, A (2003) : Utilité, utilisabilité, acceptabilité : interpréter les relations entre trois dimensions de l'évaluation des EIAH, in C. Desmoulins, P. Marquet et D. Bouhineau (Eds.), Environnements informatiques pour l'apprentissage humain, Paris, ATIEF/INRP, 391-402.

VARGA, R. (2005) : Dans quelles mesures l'utilisation d'un environnement numérique peut-elle contribuer à faire évoluer les modes d'accès aux savoirs? Colloque SIF « Les institutions éducatives face au numérique », MHS Paris Nord, 12 au 12 décembre 2005 :

Consultable sur Tematice : < http://archive-edutice.ccsd.cnrs.fr >

VARGA, R. (2006) : Suivi de stages sur une plate-forme pédagogique PostNuke, Monographie, Consultable sur Tematice :

$<$ http://archive-edutice.ccsd.cnrs.fr>

VARGA, R. et BLANC, N. (2003) : Expérience d'apprentissage d'une langue nouvelle, Module Initiation à la didactique du FLE, Poitiers, CNED (publication pédagogique).

VERMERSCh, P. et MAUReL, M. (dir.), (1997) : Pratiques de l'entretien d'explicitation, Paris, ESF.

\section{NOTES}

1. Il s'agit d'un portail dynamique, destiné à un travail collaboratif et disponible en open source.

2. Nous préconisons la tenue d'un journal de bord comme support de réflexion non seulement dans le cadre des stages, mais aussi dans un cours portant sur la méthodologie des écrits de synthèse en Licence Sciences de l'éducation à l'université Lille 3. La méthode de travail et l'évaluation proposées surprennent les étudiants et suscitent au premier abord des incompréhensions. En effet, au terme des 25 heures de cours consacrées à la méthodologie de ce type d'écrits, ils sont amenés à rédiger non pas une note de synthèse, mais une réflexion centrée sur leur vécu du cours. Ceux qui prennent à cœur la rédaction du journal de bord, arrivent généralement à produire un écrit réflexif d'une qualité remarquable.

3. L'adresse du portail PostNuke : <http://trg45.univ-lille1.fr/pcdai6>

4. Tous les étudiants valident leur UE par la rédaction d'un rapport de stage et par une soutenance orale.

5. Cette plate-forme comprend des outils d'information, des outils de communication synchrone et asynchrone et des outils de travail (Varga, 2006). 
6. Le Wiki est un outil hypertexte, destiné à la rédaction collaborative.

7. Il s'agit en fait d'un blog: outil de publication affichant les écrits de chaque utilisateur par ordre chronologique.

8. Les items entre crochets sont des liens vers des pages portant ce nom.

9. Le JDB propose une fonction « ajouter un commentaire ». Quant au Wiki, les pages peuvent être modifiées par tous les utilisateurs.

10. À ce propos, voir aussi le bilan de l'accompagnement, ci-dessous.

11. Les résultats d'une mini-enquête réalisée en mars 2006 auprès d'une cinquantaine d'étudiants en licence de communication corroborent ce constat car près de $80 \%$ pensent que les critères de notation du rapport sont prioritairement d'ordre formel : qualité de la rédaction, rapport soigné, clarté de l'expression. La pertinence de l'analyse n'occupe dans les représentations que la $2^{\mathrm{e}}$ place dans la hiérarchie des critères pour $75 \%$ de répondants.

12. En tout cas, c'est ce qui ressort de leurs discours croisés.

13. L'exploitation des données extérieures semble poser problème pour les étudiants dans les travaux réalisés car parfois ils ne font pas la différence entre intertextualité et plagiat. Remarquons en passant qu'une affaire de plagiat a secoué en 2004 la promotion des étudiants enquêtés : un étudiant a été conduit devant le conseil de discipline pour avoir copié-collé au mot près le rapport de stage d'une de ses camarades.

14. Consultables par elle seule.

15. Suite à notre enquête, la plate-forme a été légèrement restructurée, mais les outils Wiki et JDB restent inchangés.

16. Sept étudiants en M1 et quinze en L3. Il est à noter que cinq des sept étudiants en M1 ont déjà utilisé la plate-forme en 2005.

17. Entre le 15 mars et le 4 septembre 2006 on dénombre 62 connexions en moyenne par étudiant (contre 38 en moyenne pour la période de stages en 2005).

18. En moyenne 2,5 pages Wiki et 15 pages du JDB par étudiant (cf. tableau 1, ci-dessus). 19. L'élaboration d'un scénario pédagogique nous a été proposée dès le démarrage du projet PCDAI par certains collègues de Lille 1. L'idée a été rejetée à l'époque dans un but de co-évolution du dispositif numérique que nous avons souhaité adapter aux besoins réels des apprenants.

\section{RÉSUMÉS}

L'objectif de cet article est de montrer les intérêts et les limites d'une plate-forme collaborative PostNuke pour accompagner la réflexion des étudiants pendant leur stage. Les données issues de l'usage de la plate-forme en 2005 ainsi qu'une enquête réalisée après usage laissent apparaitre des décalages entre les intentions pédagogiques et les pratiques étudiantes: d'une part, ils résultent d'un certain nombre d'obstacles freinant l'usage d'un dispositif numérique dans le contexte donné ; d'autre part, ils sont révélateurs de contraintes et de tensions vécues par les étudiants, émergeant de discours souvent contradictoires. Les résultats très encourageants montrent, en revanche, les intérêts perçus par les étudiants et permettent d'envisager l'adaptation de la démarche pédagogique aux besoins réels avec la visée de modifier les modes d'accès aux savoirs. 